\title{
Strong expression of CXCL12 is associated with a favorable outcome in osteosarcoma
}

\author{
Daniel Baumhoer ${ }^{1,2, *}$, Jan Smida ${ }^{2,3, *}$, Stephanie Zillmer ${ }^{2,3}$, Michael Rosemann $^{4,5}$, \\ Michael J Atkinson ${ }^{4,5}$, Peter J Nelson ${ }^{6}$, Gernot Jundt ${ }^{1}$, Irene von Luettichau ${ }^{3,6, \dagger}$ \\ and Michaela Nathrath ${ }^{2,3, \dagger}$
}

\begin{abstract}
${ }^{1}$ Bone Tumor Reference Center at the Institute of Pathology, University Hospital Basel, Basel, Switzerland; ${ }^{2}$ Clinical Cooperation Group Osteosarcoma, Helmholtz Zentrum Muenchen, German Research Center for Environmental Health, Neuherberg, Germany; ${ }^{3}$ Department of Pediatrics, Technische Universitaet Muenchen and Pediatric Oncology Center, Munich, Germany; ${ }^{4}$ Institute of Pathology, Helmholtz Zentrum Muenchen, German Research Center for Environmental Health, Neuherberg, Germany; ${ }^{5}$ Institute of Radiation Biology, Helmholtz Zentrum Muenchen, German Research Center for Environmental Health, Neuherberg, Germany and ${ }^{6}$ Medical Policlinic, Ludwig-Maximilians University, Munich, Germany
\end{abstract}

\begin{abstract}
Hematogenous spread determines the outcome of osteosarcoma (OS) patients, but the pathogenesis of developing metastatic disease is still unclear. Chemokines are critical regulators of cell trafficking and adhesion, and have been reported to be aberrantly expressed and to correlate with an unfavorable prognosis and metastatic spread in several malignant tumors. The chemokine receptors CXCR4 and CXCR7 together with their common ligand CXCL12 form one of the most important chemokine axes in this context. To investigate a potential role of these chemokines in OSs, we analyzed their expression in a series of 223 well-characterized and pretherapeutic OS samples. Interestingly, we found the expression of CXCL12 and CXCR4 to correlate with a better long-term outcome and with a lower prevalence of metastases. These findings suggest a distinct role of CXCR4/CXCR7/CXCL12 signaling in the tumors of bone, as has also been previously described in acute leukemia. As many malignant tumors metastasize to bone, and tumor cells are thought to be directed to bone in response to CXCL12, OS cells expressing both CXCL12 and the corresponding receptors might be detained at their site of origin. The disruption of CXCR4/CXCR7/CXCL12 signaling could therefore be crucial in OSs for the migration of tumor cells from bone into circulation and for developing systemic disease.

Modern Pathology (2012) 25, 522-528; doi:10.1038/modpathol.2011.193; published online 16 December 2011
\end{abstract}

Keywords: chemokines; CXCR4; CXCR7; CXCL12; metastases; osteosarcoma; prognosis

Osteosarcomas (OSs) are highly aggressive neoplasms generally affecting the metaphyses of long bones in children and adolescents. ${ }^{1}$ As is seen in many other malignant tumors hematogenous spread primarily determines the patient's prognosis and explains why intense neoadjuvant and adjuvant chemotherapy protocols in addition to radical surgery benefit the outcome of patients significantly

\footnotetext{
Correspondence: Dr D Baumhoer, MD, Bone Tumor Reference Center at the Institute of Pathology, University Hospital Basel, 4031 Basel, Switzerland.

E-mail: dbaumhoer@mac.com

*The authors contributed equally to this work.

"Shared senior authorship.

Received 11 July 2011; revised 3 October 2011; accepted 3
} October 2011; published online 16 December 2011 when compared with surgery alone. ${ }^{2}$ Although 5 -year survival rates of up to $50-70 \%$ can be achieved by multimodal therapy, a large group of patients are still left with a poor prognosis due to lack of effective treatment options. ${ }^{3}$ Predicting the clinical course of OS patients can be achieved by assessing the response to chemotherapy histologically or, as our group recently proposed, by determining distinct chromosomal alterations already at the time of initial biopsy. ${ }^{4,5}$ The identification of patients that may not respond to first-line chemotherapy or will be at higher risk of developing metastases is required for a more precise treatment stratification. However, the pathogenesis of systemic spread in OS has not been elucidated.

The chemokine receptors CXCR4, CXCR7 and their common ligand CXCL12 are major regulators 
of cell trafficking and adhesion, and are thought to mediate hematogenous metastases in several types of cancer. Consequently, they were proposed as potential biomarkers of tumor behavior and as therapeutic targets. ${ }^{6}$ CXCL12 is known to be produced by osteoblasts and to act as a chemoattractant for CXCR4- and CXCR7-expressing cells. ${ }^{7,8}$ Interestingly, CXCL12 is also expressed in several OS cell lines that were shown to adhere to CXCR4-positive prostate cancer cells in vitro and, therefore, were used as a model to explain the predilection of prostate cancer to metastasize to bone. ${ }^{8}$ The CXCL12 receptor, CXCR4, is expressed by various types of human cancers or by distinct sub-populations of cancer cells including breast cancer, non-small-cell lung cancer, ovarian cancer and also OS. In most normal tissue types, however, CXCR4 is generally not expressed. ${ }^{9-11}$ CXCR7 (RDC-1) was identified in 2005 as a novel decoy receptor for CXCL12 interacting with CXCR4-CXCL12 signaling. ${ }^{12,13}$ Furthermore, CXCR7 was shown to contribute to neoangiogenesis in prostate cancer and to promote tumor cell proliferation in breast cancer and adenocarcinoma of the lung. ${ }^{14,15}$

The aim of our study was to investigate the role of CXCR4, CXCR7 and CXCL12 signaling in OS and in the context of systemic spread. For this purpose, we analyzed a well-characterized series of 223 OS samples that were obtained exclusively from patients before neoadjuvant treatment (diagnostic biopsies).

\section{Materials and methods}

\section{Tissue Samples and Patients' Characteristics}

All tissue samples were obtained from the archives of the Bone Tumor Reference Center at the University Hospital Basel and the Clinical Cooperation Group OS at the Helmholtz Zentrum Muenchen and comprised cases that were diagnosed between 1974 and 2010. Only samples from patients without prior treatment were included in the study $(n=223)$. Full patients' characteristics are presented in Table 1.

\section{Tissue Microarray Construction}

Tissue samples were fixed in buffered $4 \%$ formalin, decalcified using EDTA if required, embedded in paraffin, and used to construct tissue microarrays. Briefly, hematoxylin-eosin-stained sections were made from each selected primary block (donor blocks) to define representative tissue regions. Tissue cylinders $(0.6 \mathrm{~mm}$ in diameter $)$ were then punched from the respective regions of the donor block with the use of a custom-made precision instrument (Beecher Instruments, Silver Spring, USA). The number of punches per patient ranged from 1 to 8 (average 3.5, median 4.0). When more than one punch was obtained, the additional punches were taken from different representative
Table 1 Patients' characteristics

\begin{tabular}{lc}
\hline Parameter & Number (n) \\
\hline Sex & $208 / 223(93 \%)^{\mathrm{a}}$ \\
Male & 106 \\
Female & 102 \\
& \\
Age at diagnosis & $210 / 223(94 \%)^{\mathrm{a}}$ \\
Average & 22.9 years \\
Median & 16.3 years \\
Range & $4-88$ years \\
& \\
Localization & $212 / 223(95 \%)^{\mathrm{a}}$ \\
Femur & 103 \\
Tibia & 50 \\
Humerus & 17 \\
Fibula & 11 \\
Pelvis & 11 \\
Other & 20 \\
& \\
Metastases (lung) & $202 / 223(91 \%)^{\mathrm{a}}$ \\
Yes (total) & 89 \\
Yes (at initial diagnosis) & 24 \\
No & 113 \\
Time to metastases average & 15.6 months \\
Time to metastases median & 12.4 months \\
Time to metastases range & $0-79$ months \\
& \\
Follow-up & $214 / 223(96 \%)^{\mathrm{a}}$ \\
Average & 65 months \\
Median & 41.7 months \\
Range & $0-286.7$ months \\
Survival & $214 / 223(96 \%)^{\mathrm{a}}$ \\
Died & 79 \\
Alive & 135 \\
Response to chemotherapy & \\
Good & 57 \\
Poor & \\
\hline & \\
& \\
&
\end{tabular}

${ }^{\mathrm{a}} \mathrm{X} / 223(\mathrm{Y} \%)=$ data available in $\mathrm{X}$ cases $(\mathrm{Y} \%$ of the total number of cases).

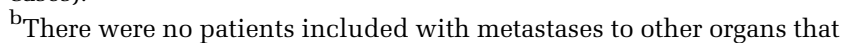
did not also have metastases to the lungs.

${ }^{\mathrm{C}}$ Good response $\leq 10 \%$ vital tumor cells in the resection specimen, poor response $\geq 10 \%$ vital tumor cells.

regions of the tumor. Tissue cylinders were transferred to two $25 \times 35 \mathrm{~mm}$ paraffin blocks (recipient blocks) to assemble the tissue microarrays. The resulting blocks were cut into $3-\mu \mathrm{m}$ sections that were transferred to glass slides by use of the Paraffin Sectioning Aid System (Instrumedics, Hackensack, USA). Subsequently, sections were used for immunohistochemistry.

\section{Immunohistochemistry}

To ensure proper immunoreactivity of tumor samples, immunohistochemistry for vimentin was performed according to routine protocols (Ventana BenchMark XT, Roche, Basel, Switzerland; CC1 pretreatment; prediluted antibody, clone V9, incubation 32 minutes at $37^{\circ} \mathrm{C}$; $\mathrm{DAB}$ chromogen). Immunohistochemistry for CXCR4 was also conducted using the Ventana BenchMark XT system 
(CC1 pretreatment; antibody from Epitomics, Roche, clone UMB2, dilution 1:500, incubation $32 \mathrm{~min}$ at $37^{\circ} \mathrm{C}$; DAB chromogen), whereas the immunoreactions for CXCR7 (microwave pretreatment in citrate buffer for $5 \mathrm{~min}$ at $70^{\circ} \mathrm{C}$; antibody from Proteintech, Lucerne, Switzerland, polyclonal, dilution 1:800, incubation over night at $4^{\circ} \mathrm{C}$; DAB chromogen) and CXCL12 (microwave pretreatment in EDTA for $20 \mathrm{~min}$ at $100^{\circ} \mathrm{C}$; antibody from R\&D Systems, Abingdon, UK, clone 79018, dilution 1:300, incubation over night at $4^{\circ} \mathrm{C}$; DAB chromogen) were performed using indirect immunoperoxidase procedures according to the manufacturer's instructions (VECTASTAIN Elite ABC Kit, Vector Laboratories, Burlingame, CA, USA). Optimal dilutions were predetermined in our laboratory.

\section{Evaluation of Immunohistochemistry}

Immunoreactivity for each protein was scored semiquantitatively by evaluating the number of positive cells over the total number of cells. Additionally, an intensity scale ranging from 0 for no, $1+$ for weak and $2+$ for strong staining was applied. All cellular compartments were evaluated separately including nuclear, cytoplasmic and membranous positivity. In cases with more than one punch per tumor the average expression was determined for further analyses. Punches that were not completely enclosed on the sections or showed artefacts due to sectioning were excluded from the analysis.

\section{Statistical Analyses}

Survival analyses were carried out using the Kaplan-Meier and Log-rank (Mantel-Cox) test. The differences in protein expression between patients with and without metastases were determined using the Mann-Whitney Test. Spearman's correlation was used to calculate the correlation between CXCR4, CXCR7 and CXCL12 expression. For multivariate survival time analysis a Cox regression model was applied. Only $P$-values $<0.05$ were considered statistically significant. All analyses were conducted using GraphPad Prism 5.0d (La Jolla, CA, USA) and SPSS 19 (IBM Corporation, Armonk, NY, USA).

\section{Results}

\section{Expression of Vimentin}

All but four cases showed strong and consistent immunoreactivity for vimentin. The four negative cases were excluded from the evaluation leaving a total of 219 OS cases for further analysis.

\section{Expression of CXCR4, CXCR7 and CXCL12}

All immunoreactions demonstrated a predominantly mixed cytoplasmic and membranous staining pattern. As a substantial fraction of cases showed strong (intensity $2+$ ) and constant ( $>90 \%$ positive tumor cells) immunoreactivity, only those cases were considered positive for the respective protein. Consequently, tumor samples with incomplete $(<90 \%$ positive tumor cells), weak or total lack of immunostaining (intensity $1+$ and 0 ) was regarded as negative (Figure 1, Table 2). In total, CXCR4 was evaluable in 159/219 (73\%), CXCR7 in 198/219 $(90 \%)$ and CXCL12 in 204/219 (93\%) cases. Drop out of samples was mainly due to cutting artefacts and/or lack of sufficient amounts of tumor tissue per punch.

\section{Correlation of Protein Expression and Patient's Survival}

The 10-year survival rate (10-YSR) differed between CXCR4-positive and -negative cases (68 vs 57\%, $P=0.1325)$ but was nearly identical between CXCR7-positive and -negative tumors (57 vs 61\%, $P=0.8158)$. Both the comparisons did not reach statistical significance. The 10-YSR between CXCL12-positive and -negative cases, in contrast, differed more distinctively (63 vs 39\%, $P=0.0068$, Figure 2) resulting in a statistically significant correlation between CXCL12 positivity and a favorable outcome. Combining CXCR4 and CXCL12 positivity yielded 10 -YSR of $71 \%$ in positive and $56 \%$ in negative cases $(P=0.0675)$.

\section{Correlation of Protein Expression and Systemic Spread}

The expression of CXCL12 was associated with a significantly lower prevalence of metastases $(P=0.02$, Table 3$)$. In case of CXCR4 and CXCR7 expression, no statistically significant correlations could be determined ( $P=0.65$ and $P=0.12$, respectively, Table 3).

\section{Correlation of Protein Expression and Response to Chemotherapy}

There were no statistically significant correlations between the expression of CXCR4, CXCR7 and CXCL12, and the response to chemotherapy $(P=0.13,0.35$ and 0.08 , respectively).

Correlation Between CXCR4, CXCR7 and CXCL12 Expression and Multivariate Survival Time Analysis

CXCL12 positivity showed a moderate positive correlation with CXCR7 $(r=0.31)$ and CXCR4 

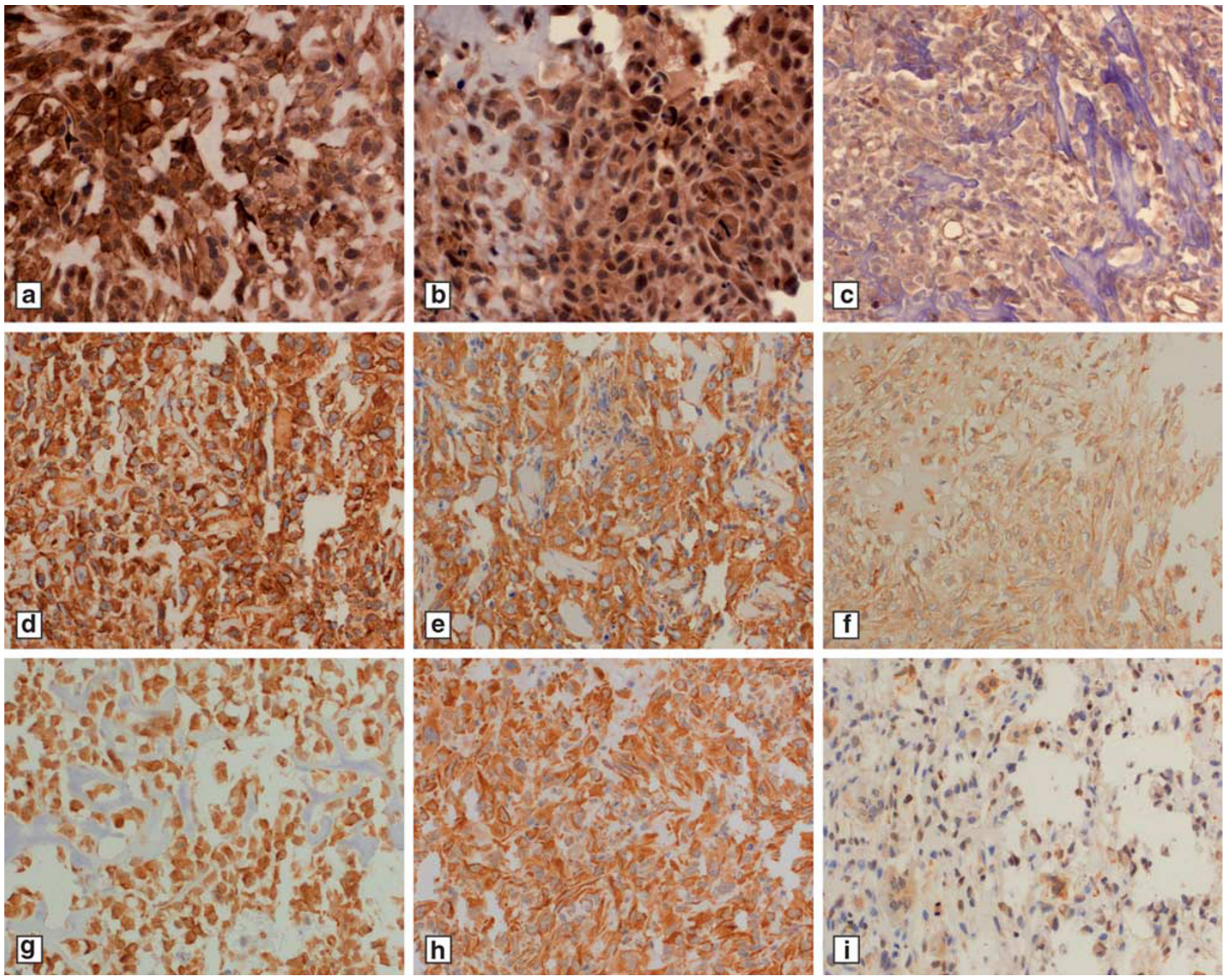

Figure 1 Immunohistochemistry for CXCR4 (a-c), CXCR7 (d-f) and CXCL12 (g-i) showed strong and constant membranous (a, d, h) and cytoplasmic (b, e, g) staining in positive cases. Weak (intensity 1+) and inconsistent staining was considered negative (c, f, i). All pictures $\times 400$.

Table 2 CXCR4, CXCR7 and CXCL12 expression

\begin{tabular}{lrcc}
\hline & Positive & Negative & Total \\
\hline CXCR4 & 78 & 81 & 159 \\
CXCR7 & 167 & 31 & 198 \\
CXCL12 & 158 & 46 & 204 \\
\hline
\end{tabular}

$(r=0.38)$ expression, CXCR4 expression correlated weakly with CXCR7 positivity $(r=0.17)$. Multivariate survival time analysis for metastases, response to chemotherapy and CXCL12 expression showed a highly significant effect only of metastases with poorer outcome (HR (95\%CI): 59.81 (8.01-446.72; $P<0.0001)$ ). Testing solely response to chemotherapy, and CXCL12 expression resulted in a significant effect only of response to therapy with clinical outcome (HR (95\%CI): 3.39 (1.71-6.74; $P<0.0001)$ ).

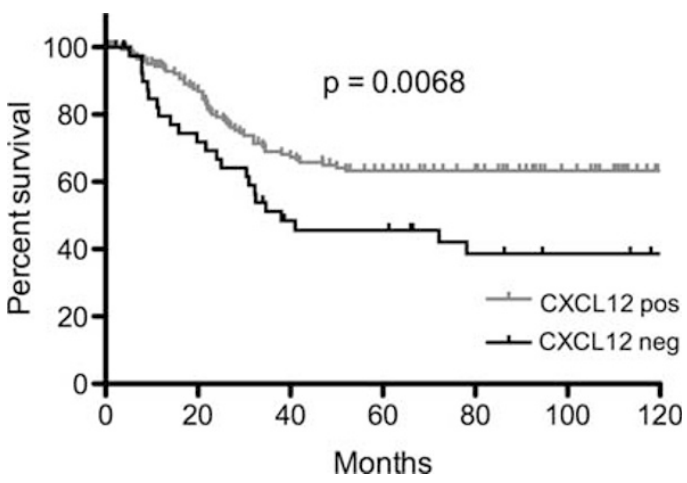

Figure 2 Kaplan-Meier curves comparing 10-year survival of osteosarcomas with and without CXCL12 expression.

\section{Discussion}

OS is a rare disease with an estimated incidence of 4-5 per million population. ${ }^{1}$ In addition to its rarity, current treatment protocols make research on OSs 
Table 3 CXCR4, CXCR7 and CXCL12 expression in cases with and without metastases

\begin{tabular}{lccrc}
\hline & Metastases & No metastases & Total & P-value \\
\hline CXCR4 positive & 29 & 42 & 71 & \\
CXCR4 negative & 33 & 41 & 74 & 0.65 \\
CXCR4 total & 62 & 83 & 145 & \\
& & & & \\
CXCR7 positive & 65 & 86 & 151 & \\
CXCR7 negative & 16 & 11 & 27 & 0.12 \\
CXCR7 total & 81 & 97 & 178 & \\
CXCL12 positive & 56 & 86 & 142 & \\
CXCL12 negative & 25 & 17 & 42 & 0.02 \\
CXCL12 total & 81 & 103 & 184 & \\
\hline
\end{tabular}

even more difficult because resection specimens have generally undergone neoadjuvant chemotherapy that can hamper molecular analyses. Therefore, only the usually scarce residual tissue from the initial diagnostic biopsies can be used for further investigations. To ensure optimal utilization of these valuable samples, tissue microarrays were constructed with a total of 223 well-characterized OSs. As the identification of novel biomarkers seems to be essential for a better treatment stratification, and for recognizing patients at high risk for systemic spread or chemoresistance, tissue microarrays represent an important tool for the analysis of large numbers of samples under the same technical conditions.

The chemokine receptors CXCR4, CXCR7 and their ligand CXCL12 have been shown to be aberrantly expressed in several malignant tumors and to be correlated with hematogenous spread and patient's prognosis..$^{8,9,14,15}$ Metastases to the bone are thought to be strongly influenced by CXCL12 signaling, as CXCL12 is known to be abundantly expressed by osteoblasts and bone marrow stromal cells. ${ }^{7,16}$ In fact, also in several OS cell lines CXCL12 expression has been reported that was utilized in experimental models to demonstrate their adhesion to CXCR4positive prostate cancer cells. The authors concluded the CXCR4/CXCL12 axis to decisively contribute to the predilection of prostate cancer to metastasize to bone. ${ }^{8}$ A recent study, furthermore, demonstrated that ursolic acid can downregulate CXCR4 expression and inhibit distant organ metastasis in a transgenic mouse model of prostate cancer. ${ }^{17}$ Interestingly, CXCR4 is known to be also expressed in several OS cell lines and has been reported to critically influence cell migration via CXCR4/CXCL12 interaction. ${ }^{10,11}$ CXCR7 is another receptor for CXCL12 with at least partial decoy function that is thought to help moderate CXCR4/CXCL12 signaling. Although its precise function is still awaiting further elucidation, several studies indicate an important impact of CXCR7 on proliferation, vascularization and metastatic potential of breast, lung and prostate cancer cells. ${ }^{14,15}$

The role and function of CXCR4, CXCR7 and their common ligand CXCL12 in OSs has been analyzed in several smaller studies using different techniques. Although CXCR7 expression has been shown to occur in OS cell lines, studies investigating this receptor on OS tissue samples and correlations to clinicopathological parameters like systemic spread and patient's prognosis have not been reported yet. ${ }^{18}$ The expression of CXCR4, however, has been analyzed at the protein and mRNA level by several groups. Oda et $a l^{19}$ reported CXCR4 to be less commonly expressed in a series of 30 OSs compared with the corresponding lung metastases of the same patients using immunohistochemistry (33 vs 67\%). Although they were not able to demonstrate a correlation between protein expression and patient's survival, they concluded CXCR4 expression to be associated with metastatic progression. A more recent study by Lin et $a l^{20}$ showed immunohistochemical CXCR4 expression in 39/56 (70\%) OS samples to correlate with a shorter survival and also with metastatic progression. In our study, we found 78/159 (49\%) CXCR4-positive cases regarding only strong and constant immunoreactivity as positive. Interestingly, CXCR4 positivity showed a trend towards a favorable long-term outcome in our series (10-YSR 68 vs $57 \%$ in negative cases, $P=0.1325$ ). Our results are, however, not easy to compare with the previous reports as both the studies used different antibodies and did not describe what kind of decalcification was conducted, which can potentially alter the immunoreactivity of tissue samples. ${ }^{19,20}$ Moreover, while Oda et $a l^{19}$ did not define the cellular compartments evaluated, Lin et $a 1^{20}$ investigated only nuclear and cytoplasmic positivity. As functional chemokine receptors would be expected on the surface of cells, we explicitly also analyzed membranous staining in our series. Although we detected nuclear CXCR4 immunoreactivity in a smaller subset of cases $(18 / 159,11 \%)$, we were not able to demonstrate a correlation to patient's survival or hematogenous spread (data not shown). Concerning the transcriptional level, our group and others reported CXCR4 mRNA to be expressed even more constantly in microdissected OS tissue samples and cell lines, suggesting this chemokine receptor to be frequently expressed in OSs. ${ }^{10,21,22}$ CXCL12 has only once been investigated immunohistochemically in a larger series of OSs and was found to be positive in just 4/113 (4\%) of cases. ${ }^{23}$ In contrast, we detected strong and constant immunoreactivity in 158/204 (78\%) of cases in our series. Li et $a l^{20}$ applied the same antibody used in our study, and although they did not state what kind of decalcification was performed or which cellular compartments were evaluated, this marked difference cannot be explained unequivocally. In our series, however, we found CXCL12 positivity to correlate with a better long-term survival (63 vs $39 \%, P=0.0068)$ and with a lower prevalence of metastases $(P=0.02)$. The multivariate survival time analysis did not suggest CXCL12 expression to represent an independent prognostic factor in our 
series but confirmed the occurrence of metastatic spread and the response to chemotherapy as the crucial prognostic factors in OS. Nevertheless, CXCL12 negativity was correlated with metastatic spread and might therefore indirectly contribute to the strong prognostic value of metastatic disease.

As most previous studies have found the expression of CXCR4 to correlate with an unfavorable outcome and systemic spread, our findings appear rather unexpected. However, in tumors originating in the bone marrow, CXCR4/CXCL12 interaction may help detain the neoplastic cells from systemic spread. In acute lymphoid and acute myeloid leukemia, CXCR4 and its ligand are thought to be responsible for the retention of leukemic cells in the bone marrow. ${ }^{24,25}$ Consequently, the disruption of CXCR4/CXCL12 signaling is thought to be a prerequisite for these cells to egress from bone marrow into circulation. ${ }^{26,27}$ OSs, however, are tumors of bone and are most likely derived from mesenchymal stem cells differentiating towards an osteoblastic lineage. As osteoblasts and OS cells have been shown to express CXCR4, CXCR7 and CXCL12, this specific chemokine axis could have a similar effect in OSs and prevent tumor cells from entering the circulation. ${ }^{7,18,28}$ Certainly, these chemokine receptors and their common ligand are not the sole regulators of hematogenous spread but could have crucial impact on the development of metastatic disease.

\section{Acknowledgements}

Daniel Baumhoer and Gernot Jundt were supported by the Foundation for the preseveration of the Basel Bone Tumor Reference Center. Jan Smida and Michaela Nathrath are members of the Translational Sarcoma Research Network supported by the BMBF. Michaela Nathrath and Irene von Luettichau were, furthermore, supported by the Wilhelm SanderStiftung within the Therapieeinheit Knochen- und Weichteilsarkome. Stephanie Zillmer was funded by the Helga und Heinrich Holzhauer Stiftung.

\section{Disclosure/conflict of interest}

The authors declare no conflict of interest.

\section{References}

1 Raymond AK, Ayala AG, Knuutila S. Conventional osteosarcoma In: Fletcher CDM, Unni KK, Mertens F (eds). World Health Organization Classification of Tumours: Pathology and Genetics of Tumours of Soft Tissue and Bone. IARC Press: Lyon, France, 2002, pp 264-270.

2 Link MP, Goorin AM, Miser AW, et al. The effect of adjuvant chemotherapy on relapse-free survival in patients with osteosarcoma of the extremity. N Engl J Med 1986;314:1600-1606.

3 Bielack SS, Kempf-Bielack B, Delling G, et al. Prognostic factors in high-grade osteosarcoma of the extremities or trunk: an analysis of 1,702 patients treated on neoadjuvant cooperative osteosarcoma study group protocols. J Clin Oncol 2002;20:776-790.

4 Salzer-Kuntschik M, Delling G, Beron G, et al. Morphological grades of regression in osteosarcoma after polychemotherapy - study COSS 80. J Cancer Res Clin Oncol 1983;106(Suppl):21-24.

5 Smida J, Baumhoer D, Rosemann M, et al. Genomic alterations and allelic imbalances are strong prognostic predictors in osteosarcoma. Clin Cancer Res 2010;16:4256-4267.

6 Sun X, Cheng G, Hao M, et al. CXCL12 / CXCR4 / CXCR7 chemokine axis and cancer progression. Cancer Metastasis Rev 2010;29:709-722.

7 Jung Y, Wang J, Schneider A, et al. Regulation of SDF-1 (CXCL12) production by osteoblasts; a possible mechanism for stem cell homing. Bone 2006;38:497-508.

8 Taichman RS, Cooper C, Keller ET, et al. Use of the stromal cell-derived factor-1/CXCR4 pathway in prostate cancer metastasis to bone. Cancer Res 2002;62:1832-1837.

9 Balkwill F. Cancer and the chemokine network. Nat Rev Cancer 2004;4:540-550.

10 Huang CY, Lee CY, Chen MY, et al. Stromal cellderived factor-1/CXCR4 enhanced motility of human osteosarcoma cells involves MEK1/2, ERK and NFkappaB-dependent pathways. J Cell Physiol 2009; 221:204-212.

11 Perissinotto E, Cavalloni G, Leone F, et al. Involvement of chemokine receptor 4/stromal cell-derived factor 1 system during osteosarcoma tumor progression. Clin Cancer Res 2005;11:490-497.

12 Balabanian $\mathrm{K}$, Lagane $\mathrm{B}$, Infantino $\mathrm{S}$, et al. The chemokine SDF-1/CXCL12 binds to and signals through the orphan receptor RDC1 in T lymphocytes. J Biol Chem 2005;280:35760-35766.

13 Burns JM, Summers BC, Wang Y, et al. A novel chemokine receptor for SDF-1 and I-TAC involved in cell survival, cell adhesion, and tumor development. J Exp Med 2006;203:2201-2213.

14 Miao Z, Luker KE, Summers BC, et al. CXCR7 (RDC1) promotes breast and lung tumor growth in vivo and is expressed on tumor-associated vasculature. Proc Natl Acad Sci USA 2007;104:15735-15740.

15 Wang J, Shiozawa Y, Wang Y, et al. The role of CXCR7/ RDC1 as a chemokine receptor for CXCL12/SDF-1 in prostate cancer. J Biol Chem 2008;283:4283-4294.

16 Dar A, Kollet O, Lapidot T. Mutual, reciprocal SDF-1/ CXCR4 interactions between hematopoietic and bone marrow stromal cells regulate human stem cell migration and development in NOD/SCID chimeric mice. Exp Hematol 2006;34:967-975.

17 Shanmugam MK, Manu KA, Ong TH, et al. Inhibition of CXCR4/CXCL12 signaling axis by ursolic acid leads to suppression of metastasis in transgenic adenocarcinoma of mouse prostate model. Int $\mathrm{J}$ Cancer 2011;129:1552-1563.

18 Van Rechem C, Rood BR, Touka M, et al. Scavenger chemokine (CXC motif) receptor 7 (CXCR7) is a direct target gene of HIC1 (hypermethylated in cancer 1). J Biol Chem 2009;284:20927-20935.

19 Oda Y, Yamamoto H, Tamiya S, et al. CXCR4 and VEGF expression in the primary site and the metastatic site of 
human osteosarcoma: analysis within a group of patients, all of whom developed lung metastasis. Mod Pathol 2006;19:738-745.

20 Lin F, Zheng SE, Shen Z, et al. Relationships between levels of CXCR4 and VEGF and blood-borne metastasis and survival in patients with osteosarcoma. Med Oncol 2011;28:649-653.

$21 \mathrm{Kim}$ SY, Lee CH, Midura BV, et al. Inhibition of the CXCR4/CXCL12 chemokine pathway reduces the development of murine pulmonary metastases. Clin Exp Metastasis 2008;25:201-211.

22 von Luettichau I, Segerer S, Wechselberger A, et al. A complex pattern of chemokine receptor expression is seen in osteosarcoma. BMC Cancer 2008;8:23.

23 Li Y, Flores R, Yu A, et al. Elevated expression of CXC chemokines in pediatric osteosarcoma patients. Cancer 2011;117:207-217.
24 Juarez J, Dela Pena A, Baraz R, et al. CXCR4 antagonists mobilize childhood acute lymphoblastic leukemia cells into the peripheral blood and inhibit engraftment. Leukemia 2007;21:1249-1257.

25 Nervi B, Ramirez P, Rettig MP, et al. Chemosensitization of acute myeloid leukemia (AML) following mobilization by the CXCR4 antagonist AMD3100. Blood 2009;113:6206-6214.

26 Teicher BA, Fricker SP. CXCL12 (SDF-1)/CXCR4 pathway in cancer. Clin Cancer Res 2010;16:2927-2931.

27 Tavor S, Petit I. Can inhibition of the SDF-1/CXCR4 axis eradicate acute leukemia? Semin Cancer Biol 2010;20:178-185.

28 Zhu W, Liang G, Huang Z, et al. Conditional inactivation of CXCR4 in osteoprecursors reduces postnatal bone formation due to impaired osteoblast development. J Biol Chem 2011;286:26794-26805. 